“C 2017 IEEE. Personal use of this material is permitted. Permission from IEEE must be obtained for all other uses, in any current or future media, including reprinting/republishing this material for advertising or promotional purposes, creating new collective works, for resale or redistribution to servers or lists, or reuse of any copyrighted component of this work in other works." 


\title{
Internet of Things (IoT) in E-commerce:
}

\author{
For People with Disabilities
}

\author{
Osama Sohaib \\ Faculty of Engineering and IT \\ University of Technology Sydney \\ Australia \\ Osama.Sohaib@uts.edu.au
}

\author{
Haiyan Lu \\ Centre for Artificial Intelligence \\ Faculty of Engineering and IT \\ University of Technology Sydney \\ Australia \\ Haiyan.Lu@uts.edu.au
}

\author{
Walayat Hussain \\ Faculty of Engineering and IT \\ University of Technology Sydney \\ Australia \\ Walayat.Hussain@uts.edu.au
}

\begin{abstract}
Internet of Things (IoT) is an interconnection between the physical object and digital world. As a result, many e-commerce companies seize the advantages of the IoT to grow their business. However, the world's largest minority are people with disabilities. IoT can lower barriers for the disabled people by offering assistance in accessing information. Increasing Internet accessibility can help to make that happen for both social and economic benefit. This paper presents the proposed integrated framework of the IoT and cloud computing for people with disabilities such as sensory (hearing and vision), motor (limited use of hands) and cognitive (language and learning disabilities) impairments in the context of business-to-consumer e-commerce context. We conclude that IoT-enabled services offer great potential for success of disabled people in the context of online shopping.
\end{abstract}

Keywords- Accessibility; E-commerce; Internet of Things (IoT); Cloud Computing, Disabled People

\section{INTRODUCTION}

An e-commerce website is the central way an e-retailer communicates with their online consumers. E-retailer seeks to provide positive online purchasing experiences for online consumers of all ages. According to the World Health Organisation [1], billion people in the world, which is $15 \%$ of the total population has disability that limits their participation in the community. People with disabilities have limitations for going shopping, which put them at inconvenience because of their physical handicap. However, disabled people can gain a sense of emotional stability by online shopping. Therefore, websites need to be accessible to all consumers, including those with disabilities. Though, if e-commerce websites are inaccessible, consumer with disabilities do not have the equal access they are guaranteed by law.

Many online consumers may have various types of disabilities, such as sensory (hearing and vision), motor (limited use of hands) and cognitive (language and learning disabilities) impairments. Because of the Internet availability, online shops provide consumer the ease of buying and selling products. Inclusion of people with disabilities and the aged in mainstream society has improved significantly through the continuous development of guideline and assistive technologies together with the use of Information and Communications Technology (ICT). Such as World Web Content Accessibility Guidelines (WCAG) developed by Wide Web Consortium (W3C) have to reduce the barrier for accessing information. WCAG provides recommendations to make web content more accessible for user of all ages and with disabilities. For that reasons, the Web Content Accessibility Guidelines (WCAG 2.0) [2] helps to make the website accessible for users of all ages and with disabilities such as color blindness, deaf users, and age related vision problems. Though, In [3] the authors found that $78.9 \%$ of the webmasters were aware that there are automatic tools to check web accessibility. This means that the missing knowledge is not the main reason for the absence of development of accessible websites. The required web technological infrastructure is either insufficient or does not exists in terms of web accessibility for disabled people [4]. Web accessibility is particularly important since blind or color blind consumer have much more difficulty browsing the web [5]. The accessibility evaluation in e-commerce has been limited, in particular for disabled people in the context of business-toconsumer (B2C) consumer.

One new technological trend that will impact on society (and thus people with disabilities and the aged) is that of the Internet of Things (IoT) [6]. IoT is the interconnection of every physical thing (digital objects) that is networked smart devices [7]. The smart devices are able to understand, sense and respond to the environment through Internet and Radiofrequency Identification (RFID), context awareness or embedded sensors technologies [8]. This technological development of IoT enables new ways of communication between smart objects and people and between objects themselves [9].

It is highly desirable that e-commerce firms should make 
greater efforts to ensure that the consumers with disabilities have equal access to online shopping. The IoT can enable people with disabilities the support they need to attain a good quality of life and allows them to participate in the social life. Therefore, the purpose of this paper is to analyze how people with sensory (hearing and vision), motor (limited use of hands) and cognitive (language and learning disabilities) impairments can benefit from the IoT in the context of online shopping [10]. This study presents the proposed integrated framework of the IoT and cloud computing for people with disabilities such as sensory (hearing and vision), motor (limited use of hands) and cognitive (language and learning disabilities) impairments in the context of business-toconsumer e-commerce context.

\section{BACKGROUND AND RELATED STUDIES}

This section discusses some background information and related studies about IoT, cloud computing and electronic product code. We studied several researches focused on, how to connect objects (things) to produce accessible information.

\section{A. Barriers for Consumer with Disabilities in E-Commerce}

The following examples may help to explain the types of barriers that can be encountered by an online consumer with a disability.

- Visual objects. Product images are placed on the ecommerce website. Websites that use flashing images could trigger symptoms for those with seizure disorders [11]. These images are an example of a barrier for consumers who are visually impaired. They are unable to see the product image and therefore unable to buy it. Such as the use of screen readers cannot read images, animations, navigational buttons, as well as some difficulties with reading layout tables and charts [12]. In addition, a color-blind consumer making online purchase will not differentiate the red font highlighting the discounted prices.

- Audio objects. An e-retailer has recorded product information available to consumer on the website as an audio clip. Also, other products such as digitized audio. Then consumer who has difficulty in hearing or deaf cannot hear to buy the product.

- Language difficulty. If the product information displayed on the website page is written in unnecessarily complicated language, such as the use of technical terms in specifications of electronics products. Then it may present serious difficulty for consumers with language, learning or cognitive disabilities (for-example, reading disabilities, thinking, remembering, sequencing disabilities). In addition, all consumers can face comprehension barriers.

- Objects Interaction. When an e-retailer focuses on more advanced image interactivity technology, such as three-dimensional (3D) virtual models, 360-degree rotation view allows the manipulation of product images. These will represent a barrier for a consumer who cannot grip the mouse to imitate actual experiences with the product. For-example, consumers with various forms of motor impairments may have increased difficulty using a mouse or keyboard. Such as, rollovers and drop- down menus are difficult to use without a mouse.

\section{B. Internet of Things (IoT)}

The basic idea of IoT is to connect a variety of things around us to interact with each other, For-example RadioFrequency IDentification (RFID) tags, sensors, actuators, tablets and smart phones etc. The work by [13] provided an overview of the IoT architecture for people with disabilities. It is divided into three main layers: (1) Perception layer: This layer is concerned to identify objects and collect information through mainly by sensors and actuators, smart devices (smart phone, tablets etc.) and RFID tags and readers etc. (2) Network layer: as the name suggest this layer made up of wired/wireless privately owned networks or Internet etc. The main purpose of this layer to communicate information obtained from the perception layer. (3) Application layer: this layer applies the IoT technologies for providing solutions to satisfy user needs. In [14], the authors discussed IoT technology applications and their integration in e-commerce inventory, logistics and online payment. In [15] and [16], the authors propose systems to help visually impaired people in brick and mortar shopping for product identification and search. The RFID database converts the scan tag or barcode into human- interpretable product information and returns it to the handheld computer or smart device. In addition, [17] proposed real time product detection using actual video stream. A consumer composes a shopping list of items on the website and uploads it on a mobile. The audio was later used in the shop to guide the shopper. Industry experts agree that the IoT has a great potential to bring benefits to people with disabilities [18].

\section{Cloud Computing}

The Internet of Things (IoT) is tightly coupled in the cloud computing. Cloud computing is a model for big data storage, visualization, computation and analytics etc. The integration of cloud/mobile cloud computing and IoT and has great potential for success in e-commerce. Mobile cloud computing is similar to cloud computing, where data storage takes place in the cloud through wireless networks by mobile devices [19].

Cloud Computing includes three different service models, which are Platform-as-a-Service (PaaS), Infrastructure-as-aService (IaaS) and Software-as-a-Service (SaaS) [19]. SaaS are used to consume the services; it provides end-user to run applications on a cloud that is mainly accessed via web service technologies. IaaS are used to host the services, such as provision of hardware, servers, data storage and networking components to users. PaaS is used to build the service, such as a developer want to develop and run a cloud application for a 
particular purpose. In the context of B2C e-commerce, SaaS platform allows online business solution.

The integration of IoT and cloud computing can enable smart devices of sensing data stream. Such as, the cloud computing can allow stored sensing data to be used intelligently for smart monitoring and actuation with the smart devices. A cloud computing can provide the virtual infrastructure for utility computing integrating applications. Cloud computing that offers utility-based model will enable businesses and users to access applications on demand anytime, anyplace and anywhere [20]. For example, in the context of e-commerce for disable consumer the cloud system will transform data using smart devices into insight and drive productive actions from these insights. In [21], the authors presented a cloud implementation using Aneka (which is based on private and public clouds interaction) to provide the capacity for utilizing the IoT.

\section{IoT Technologies}

Mature technologies such as Near Field Communication (NFC) enables technologies such as reminder assistant widget and other assistive functions like electronic product tag, NFCbased pairing and trust verifications. Bluetooth low energy (BLE) allows devices communicate with other parts in this framework robustly with less power consumption. Furthermore, with Bluetooth class 1 compatible devices, a 100-meters communication distance is possible. Other functions such as PrimeSense System on a Chip (SoC) uses 3 imensional human body movement detection and sensing allows users to use gestures or body movements to interact with the e-commerce system or give commands. Long Term Evolution (LTE) is a mobile broadband technology makes real-time heavy load network transmission possible in a mobile IoT device with low power consumption.

Electronic product code (EPC) has become one of the main research interests for academics and also in several industries in recent years [8]. The increasing diffusion of the EPC was first proposed by the Auto-ID Center, founded in 1999 at Massachusetts Institute of Technology (MIT) [22]. The AutoID project ensures Radio-Frequency IDentification (RFID) data interoperability in enterprise wide applications. The EPC is considered as a unique numbering structure to detect all types of physical objects. The purpose of EPC technology is to create an IoT that consist of various components (hardware and software) including open-standards to bridge the gap between the physical and virtual worlds [23].

Radio-frequency Identification (RFID) technology is a major development in the embedded communication paradigm to help in the automatic identification of anything they are attached to acting as an electronic barcode [24] The passive RFID tags communicate the ID to the RFID reader by using the power of the reader's interrogation signal that is not battery powered. This has resulted in many enterprise wide applications such as in retail and supply chain management. [8] developed eCloudRFID framework for mobile devices to enable the development process of the integration process of
EPC network instances and embedded RFID and business applications. The authors presented mobile RFID services for the IoT, whcih can be made using RFID in smart devices.

EPC network is a conceptual model that enables RFID information sharing between parties of any business. The technical development of the EPC framework of components is specified by EPCglobal [25]. Figure 1 presents the EPC network infrastructure. This architecture is composed of the following elements (top-bottom): Enterprise Applications, Information Service Layer, Object Name Service (ONS), and Discovery Service (DS), Middleware Layer and the Tags, Reader Devices. The Information Service layer is an information repository to save and access the read data about items identified by tag reader through the middleware. The middleware is a software layer that aims to improve the communication interface between RFID readers and application clients. On the other hand, ONS is a similar concept to domain name service (DNS) used in the Internet to show which web server has the information related to an object. DS is a set of services that permits users to access the information related to specific objects [23]. This layer is important because it is responsible for filtering of large volume of read data generated by tag and readers devices for avoiding data duplicity [26].

Fig 1. Components of the EPC Network [8].

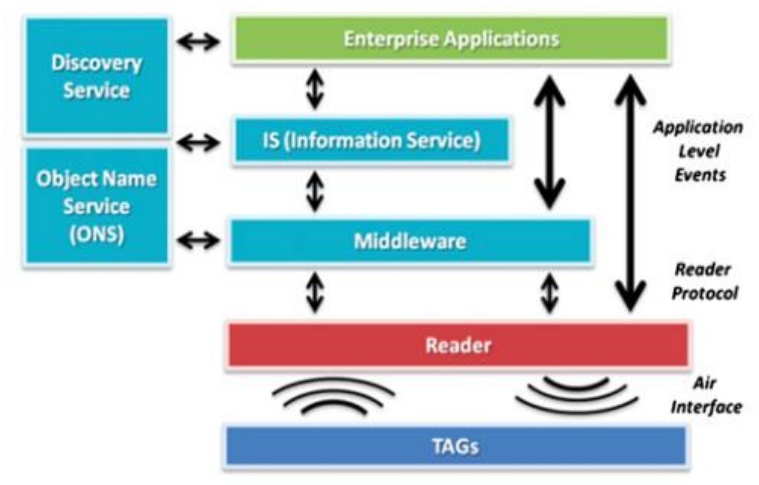

\section{IOT BASED E-COMMERCE FRAMEWORK}

People with disabilities and aged can now use improved accessibility features in smart devices (such as smartphones/tablets etc.). Many manufacturers have developed smart devices with easier user interfaces. These user interfaces enable use by disable users whose usage of smart devices may otherwise be affected by vision or hearing loss or reduced mobility. These accessible smart devices are already in use for smart cities, smart homes, smart transportation or e-health, giving disable person a new and flexible control to participate more fully in the economic environment. In [27], the authors presented an IoT architecture stack for disabled people.

We propose an integrated IoT and cloud computing application to enable the creation of smart devices (such as smartphone or tablets) to be able to support a large number of 
disabled people for online shopping in a reliable manner. The proposed integrated cloud and IoT conceptual framework is shown in Figure 2.
Fig 2. Integrated Conceptual IoT and Cloud framework.

Smart devices are able to work in wireless network environments without the fear of limitations such as access

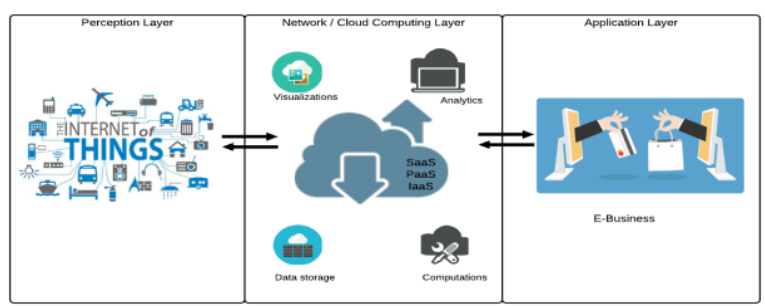

devices or data sources with limited power and unreliable connectivity. In particular, we propose an iAccess service to provide the user-driven service modeling process. A conceptual framework integrating the IoT sensing devices, cloud computing and the applications are shown in Figure 3.

Fig 3. Proposed framework on online shopping scenario for disabled users.

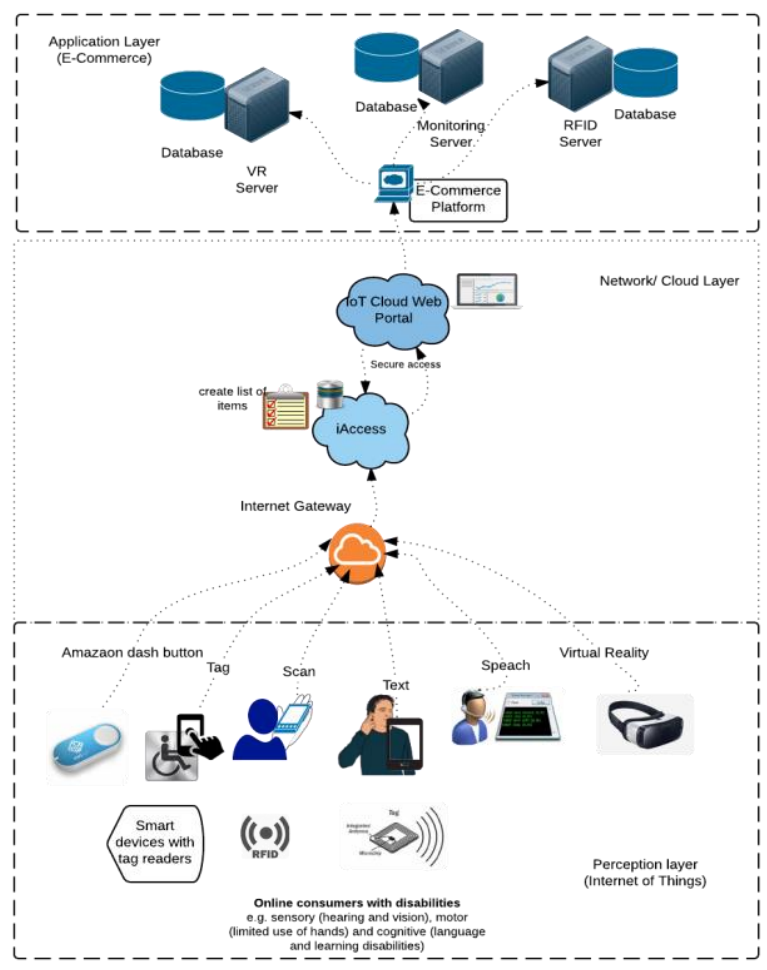

The cloud based iAccess service will allow a disable consumer to compiles a digital shopping list of item(s) using a speech recognition or text, scanning the barcode or using RFID reader through smart device and deliver it to IoT cloud portal. The created list of item(s) is stored and registered in the service repository to be detected in the IoT cloud based service web portal. The IoT service abstracts the service modeling result to deliver to the e-commerce service platform. 
The web portal will display the required item to the user. Online payment can also be completed using RFID. Such as RFID chips embedded in smart devices or RFID credit cards.

In addition, Amazon Dash Button has been a great improvement of convenience for ordering household routinely used products like laundry powder, coffee, tissue, toothpaste etc. Once the button is pressed, it will send a notice to your smartphone and ask for confirmation of the order [28]. Furthermore, Virtual Reality (VR) is described as immersive environments responsive to the actions of the user, providing opportunities for repetitive, contextual practice and feedback consistent with conditions for successful motor skill acquisition [29]. With the deployment of virtual experience, user will also feel more involved in the online shopping activities. In general, VR system has an edge in helping people with disabilities, engage user (especially older people) to be more active (in improving health-related domain), and even provide companies with more ways to communicate with their customers. Virtual experience will also potentially create a cognitively engaging experience (Liu \& Shum 2002). Comparing with a graphic representation of a product, an interactive 3-D experience in a product interface tends to foster the formation of a more accessible and confident brand attitudes. Additionally, PrimeSense 3D Sensors technology based on gesture recognition enables natural interaction between people and smart devices and between devices and their surroundings. This technology has the potential for online shopping. For-example, "Shopperception uses PrimeSense technology to provide brick-and-mortar retailers with an amazingly advanced system for understanding precisely and in real-time just what shoppers do in front of a retail shelf" [30].

\section{CONCLUSION}

The main strength of the IoT is the high influence it will have on several aspects of everyday-life and behavior of disabled people. IoT creates an integral part of future Internet. On the other hand, cloud computing is a more mature technology offering several advantages. We propose a framework of the integration of IoT and cloud computing to enable great potential for success of disabled people in the context of e-commerce. The proposed framework is an environment aimed at lowering barriers for the disabled people. Increasing Internet accessibility can help to make that happen for both social and economic benefit.

E-commerce firms need to make accessibility a priority for disabled people in their online store. This study is intended as a starting point for the e-commerce firms to work together to make change for people with disabilities. Online store manager can use the proposed framework in this study to increase the chances for an online business to succeed globally with diverse degrees of Internet users. Practical implications extend to business firms to make changes to their online business strategies to trigger their online sale better by targeting consumers with disabilities.
In future work, the implementation of our proposed framework is required to ensure accessible e-commerce for people with disabilities. In addition, IoT technology can be used in various aspect of e-commerce such as the development of Augmented Reality (AR) in our proposed framework on smart devices for users of all ages and with disabilities is a big motivation.

\section{REFERENCES}

[1] WHO, "Disabilities and rehabilitation," 2014.

[2] W3C. (2005, 18 May 2012). Introduction to Web Accessibility. Available: http://www.w3.org/WAI/intro/accessibility.php

[3] J. Lazar, D. Alfreda, and K. Greenidge, "Improving web accessibility: a study of webmaster perceptions," Computers in Human Behavior, vol. 20, pp. 269-288, 2004.

[4] O. Sohaib and K. Kang, "Individual level culture influence on online consumer iTrust aspects towards purchase intention across cultures: A S-O-R model," International Journal of Electronic Business, vol. 12, pp. 142-161, 2015.

[5] C. Brunsman-Johnson, S. Narayanan, W. Shebilske, G. Alakke, and S. Narakesari, "Modeling web-based information seeking by users who are blind.," Disability and Rehabilitation: Assistive Technology, , vol. 6, pp. 511-525., 2011.

[6] M. Chui, M. Löffler, and R. Roberts. 2010, The Internet of Things. Available:

http://www.mckinseyquarterly.com/High_Tech/Hardware/The_Internet _of_Things_2538?gp=1

[7] J. Pascual, O. Sanjuá n, J. M. Cueva, B. C. Pelayo, M. A'lvarez, and A. Gonza'lez, "Modeling architecture for collaborative virtual objects based on services.," Journal of Network and Computer Applications vol. 34, pp. 1634-1647, 2011.

[8] L. A. Amaral, F. P. Hessel, E. A. Bezerra, J. C. Corrêa, O. B. Longhi, and T. F. O. Dias, "eCloudRFID - A mobile software framework architecture for pervasive RFID-based applications," Journal of Network and Computer Applications, vol. 34, pp. 972-979, 2011.

[9] L. Tan and N. Wang, "Future internet: the internet of things. ," in Proceedings of third international conference on advanced computer theory and engineering, 2010.

[10] O. Sohaib and K. Kang, "Internet of Things (IoT) in E-Commerce for People with Disabilities," presented at the The 15th International Conference on Electronic Business, Hong Kong, 2016.

[11] N. Golden, "Access this: Why institutions of higher education must provide access to the Internet to students with disabilities," Journal of Entertainment and Technology Law,, vol. 10, pp. 363-411, 2008.

[12] K. L. Crow, "Four types of disabilities: Their impact on online learning," Techtrends, vol. 52, pp. 51-55, 2008.

[13] M. C. Domingo, "An overview of the Internet of Things for people with disabilities," Journal of Network and Computer Applications, vol. 35, pp. 584-596, 2012.

[14] X. Xu, "IOT Technology Research in E-commerce," Information Technology Journal, vol. 13, pp. 2552-2559, 2014.

[15] P. E. Lanigan, A. M. Paulos, A. W. Williams, D. Rossi, and P. Narasimhan, "Assistive technologies for grocery shopping for the blind," in Proceedings of the IEEE-BAIS symposium on research in assistive technologies, 2006, pp. $147-148$.

[16] J. Nicholson, V. Kulyukin, and D. Coster, "ShopTalk: Independent blind shopping through verbal route directions and barcode scans," The Open Rehabilitation Journal vol. 2, pp. 11-23, 2009. 
[17] T. Winlock, E. Christiansen, and S. Belongie, "Toward real-time grocery detection for the visually impaired," in Proceedings of the computer vision applications for the visually impaired workshop, 2010.

[18] E. Borgia, "The Internet of Things vision: Key features, applications and open issues," Computer Communications, vol. 54, pp. 1-31, 2014.

[19] K. E. Psannis, S. Xinogalos, and A. Sifaleras, "Convergence of Internet of things and mobile cloud computing, Systems Science \& Control Engineerin," Systems Science \& Control Engineering: An Open Access Journal, vol. 2, pp. 476-483, 2014.

[20] D. Canellos. (2013). How the Internet of Things will Feed Cloud Computing next Evolution. Available: HTTPS://BLOG.CLOUDSECURITYALLIANCE.ORG/2013/06/05/H OW-THE-INTERNET-OF-THINGS-WILL-FEED-CLOUDCOMPUTINGS-NEXT-EVOLUTION/

[21] J. Gubbi, R. Buyya, S. Marusic, and M. Palaniswami, "Internet of Things (IoT): A vision, architectural elements, and future directions," Future Generation Computer Systems, vol. 29, pp. 1645-1660, 2013.

[22] F. Thiesse and F. Michahelles, "An overview of EPC technology," International Journal of Sensor Review vol. 26, pp. 101-105, 2006.

[23] K. S. Leong, N. M.L, and D. W. Engels, "EPC Network architecture," Auto-ID center, 2006.

[24] E. L. Welbourne, G. Battle, K. Cole, K. Gould, S. Rector, and e. a. Raymer, "Building the Internet of Things using RFID The RFID ecosystem experience," IEEE Internet Computing, vol. 13, pp. 48-55, 2009.

[25] EPCglobal. (2015, September 2015). EPCglobal. Available: http://www.gs1.org/epcglobal

[26] C. Floerkemeier, C. Roduner, and M. Lampe, "RFID application development with the Accada Middleware Platform," System Journal vol. 1, pp. 82-94, 2007.

[27] N. V. Lopes, F. Pinto, P. Furtado, and J. Silva, "IoT architecture proposal for disabled people," in 2014 IEEE 10th International Conference on Wireless and Mobile Computing, Networking and Communications (WiMob), 2014, pp. 152-158.

[28] C. Dolan. (2016, 18 October 2016). Amazons New Programmable Dash Button. Available: http://consumersresearch.org/amazons-newprogrammable-dash-button/

[29] B. S. Lange, P. Requejo, and S. M. Flynn, "The potential of virtual reality and gaming to assist successful aging with disability," Phys Med Rehabilitation Clinic, vol. 21, pp. 339-356, 2010.

[30] I. Shel. (2013). How Walmart and Heineken Will Use Shopperception to Put Your In-store Experience in Context. Available: http://www.forbes.com/sites/shelisrael/2013/01/27/how-walmart-andheineken-will-use-shoppercetion-to-put-your-in-store-experience-incontext/\#4ed6a8dc1fb7 